\title{
Remodeling of the $C$. elegans Non-coding RNA Transcriptome by Heat Shock
}

William P. Schreiner ${ }^{1}$, Delaney C. Pagliuso ${ }^{1}$, Jacob M. Garrigues ${ }^{1}$, Jerry S. Chen ${ }^{2}$, Antti P. Aalto ${ }^{3}$, and Amy E. Pasquinelli ${ }^{* 1}$

${ }^{1}$ Division of Biology, University of California, San Diego, La Jolla, CA 92093-0349, USA

${ }^{2}$ Encoded Genomics, South San Francisco, CA 94080, USA

${ }^{3}$ ProQR Therapeutics N.V., 2333 CK Leiden, The Netherlands

Keywords: heat shock, HSF-1, C. elegans, miRNA, lincRNA, Helitron

* To whom correspondence should be addressed. Tel: +1-858-822-3006; Fax: +1-858822-3021; Email: apasquinelli@ucsd.edu 


\begin{abstract}
Elevated temperatures activate a Heat Shock Response (HSR) to protect cells from the pathological effects of protein mis-folding, cellular mis-organization, organelle dysfunction and altered membrane fluidity. This response includes activation of the conserved transcription factor Heat Shock Factor 1 (HSF-1), which binds Heat Shock Elements (HSEs) in the promoters of genes induced by heat shock (HS). The upregulation of protein-coding genes (PCGs), such as Heat Shock Proteins (HSPs) and cytoskeletal regulators, is critical for cellular survival during elevated temperatures. While the transcriptional response of PCGs to heat shock has been comprehensively analyzed in a variety of organisms, the effect of this stress on the expression of noncoding RNAs (ncRNAs) has not been systematically examined. Here we show that in Caenorhabditis elegans HS induces up- and down-regulation of specific ncRNAs from multiple classes, including miRNA, piRNA, lincRNA, pseudogene, and repeat elements. Moreover, some ncRNA genes appear to be direct targets of the HSR, as they contain HSF-1 bound HSEs in their promoters and their expression is regulated by this factor during HS. These results demonstrate that multiple ncRNA genes respond to HS, some as direct HSF-1 targets, providing new candidates that may contribute to organismal survival during this stress.
\end{abstract}




\section{INTRODUCTION}

In the natural world, animals experience a variety of potentially lethal environmental challenges. Heat stress is one of the most recognizable and can be fatal, if not properly addressed. Organisms have evolved an ancient response to cope with this dangerous insult: The Heat Shock Response (HSR) (1). Heat Shock Factor 1 (HSF-1), serves as a master transcriptional regulator of the HSR in eukaryotes (2). Elevated temperatures trigger activation and binding of HSF-1 to Heat Shock Elements (HSEs) in the promoters of genes encoding Heat Shock Proteins (HSPs) and other factors that protect the cell from heat-induced damage. For example, HSPs act as molecular chaperones to deal with the rampant protein mis-folding associated with heat stress. While heat shock has been shown to induce widespread changes in gene expression in cell as well as animal models, a limited set of genes appears to be direct targets of HSF-1 activation (3-5). Instead, downstream transcriptional effectors mediate many of the other HSinduced changes in gene expression (3-5).

There is also potential for post-transcriptional mechanisms to regulate the levels of protein-coding mRNAs during the HSR (6-9). In C. elegans, specific microRNAs (miRNAs) have been found to be up- or down-regulated during HS $(10,11)$. MiRNAs function as small, $\sim 22$ nucleotide, guide RNAs that use partial base-pairing to recruit Argonaute (AGO) proteins to specific mRNAs, triggering mRNA destabilization and translational repression (12). While direct mRNA targets of HS-regulated miRNAs are yet to be determined, loss of some of these miRNAs has been shown to affect the viability of $C$. elegans subjected to HS $(11,13)$. For example, deletion of miR-71 or miR239 results in reduced or enhanced survival at elevated temperatures, respectively.

In addition to miRNAs, the expression of other types of non-coding RNAs (ncRNAs) can respond to fluctuations in environmental conditions $(14,15)$. The $C$. elegans long ncRNA, rncs-1 (RNA non-coding starvation up-regulated) is induced by food deprivation and potentially regulates other small RNA pathways (15). In a variety of organisms, HS and other stress conditions have been shown to cause accumulation of RNAs from transposon and other types of repetitive sequences (16). Currently, it is unclear if the HS-induced changes in ncRNA expression are a direct result of the HSR or the aftermath of defective transcriptional or post-transcriptional silencing mechanisms under these conditions. Additionally, whether specific ncRNAs or generally all members of a certain class respond to HS has not been systematically investigated.

To examine the organismal response of different classes of ncRNAs to HS, we surveyed the expression of miRNAs, Piwi RNAs (piRNAs), long intergenic ncRNAs (lincRNAs), ncRNAs, pseudogene- and repeat-derived RNAs in C. elegans under HS versus control temperature conditions. Within each class, we observed HS-induced changes for specific transcripts that included rapid and dramatic up-regulation of some ncRNAs. Similar to canonical HS-responsive protein-coding genes, we found that the promoter sequences of the miRNA miR-239, Helitron1_CE transposons, and the pseudogene dct-10 contain Heat Shock Elements (HSEs) that are bound by HSF-1 in response to HS. Furthermore, we show that up-regulation of these ncRNAs in HS is 
regulated by HSF-1, suggesting that they are direct transcriptional targets of the HSR. Overall, our comprehensive analysis of ncRNA expression in response to HS revealed new types of molecules that are regulated by and, in turn, may contribute to organismal survival in the face of this stress.

\section{MATERIALS AND METHODS}

\section{Sequencing and analysis of mRNAs and long ncRNAs}

$\mathrm{N} 2$ wild-type worms were grown to $\mathrm{L} 4$ stage in a $20^{\circ} \mathrm{C}$ incubator under standard growth conditions (17). The experimental group was subjected to heat stress by raising the temperature to $35^{\circ} \mathrm{C}$ for 4 hours. Animals were then collected, snap-frozen, and total RNA was extracted using a standard Trizol RNA extraction protocol. cDNA sequencing libraries were prepared from total RNA from N2 wild-type control or heat shocked worms using the standard protocol from the Illumina Stranded TruSeq RNA library prep kit. Prior to library preparations, ribosomal RNA was removed using RiboZero Gold (Illumina). cDNA libraries were sequenced on an Illumina Genome Analyzer II (100 bp paired-end reads). FASTQ reads were first trimmed using fastq-mcf (https://expressionanalysis.github.io/ea-utils/), which removed flanking Illumina adapter sequence as well as nucleotides with low quality sequencing scores. Reads were then aligned to the $C$. elegans genome WS235 using STAR (18). Aligned reads were sorted using Samtools (19). Reads were counted using FeatureCounts and Ensembl 88 gene annotations (20). Differential expression of gene expression was determined using DESeq2 (21). Pseudogenes, lincRNAs, and ncRNAs are included in the Ensembl 88 gene annotations. After differential expression, these classes of genes were filtered out of the results and analyzed separately. See github.com/wschrein for code and additional example graphs.

To identify false positive up-regulated mRNAs that likely resulted from failure of Pol II transcriptional termination of an upstream HS-responsive gene, we used a strategy similar to that in Duarte et al., 2016 (3). First, an intron retention score (IR score) for each gene was calculated by dividing the total normalized intron reads by the total normalized exon reads per gene. Reads were normalized by DESeq2 which normalizes to sequencing depth but not length. Next, up-regulated genes were analyzed for accumulation of Intergenic Junction (IJ) reads between their annotated start site and the closest upstream gene. To do this, a 21 bp region that overlaps $11 \mathrm{nts}$ into the 5' annotated start site and $10 \mathrm{nts}$ upstream of the start site was obtained for each gene. The location for these regions was obtained by parsing a list of Intergenic regions downloaded from the WS235 version of the Wormbase ftp site (22). Reads for this region in both control and heat shock samples were obtained using the program FeatureCounts. The IJ Ratio for each gene was calculated by dividing the normalized (for depth) HS Intergenic reads by the CTRL Intergenic reads. Genes with an IR score greater than 0.4 and an IJ ratio greater than 2 were removed from the list of upregulated PCGs. In addition, PCGs with an IR score greater than 1 were also filtered out as these reads derived from independently transcribed ncRNAs, such as tRNAs and snoRNAs, present in the intron of the PCG. Finally, PCGs that overlapped a repetitive 
element by $>50 \%$ were filtered out. A list of $C$. elegans repetitive elements was obtained from the UCSC genome Browser. Overlap was determined using Bedtools intersect (23).

To analyze the expression of different classes of Repetitive Element RNAs, RNASequencing data were aligned to a set of $C$. elegans consensus repeats obtained from repbase (24). Primary aligned reads were obtained using the following command: samtools view -F 260 \$s\} | cut -f 3 | sort | uniq -c | awk '\{printf("\%slt\%sln", \$2, \$1)\}' > $\$\{$ s\}counts.txt Differential expression was determined using DESeq2. More detailed information including sample code/commands can be found on github.com/wschrein.

\section{Small RNA sequencing and analysis}

C. elegans were grown to the L4 stage at $20^{\circ} \mathrm{C}$ then shifted to $35^{\circ} \mathrm{C}$ for six hours. Animals were then collected, snap-frozen, and total RNA was extracted using a standard Trizol RNA extraction protocol. Small-RNA libraries were prepared using Illumina's TruSeq Small RNA library prep kit. miRNAs were analyzed by mapping small RNA sequencing data to $C$. elegans miRNAs. DESeq2 was used to determine differential expression from two independent biological replicates. piRNAs were analyzed by mapping small RNA sequencing data to a database of $C$. elegans piRNAs obtained from Wormbase using the STAR aligner. Primary reads were obtained using samtools, and differential expression was determined using DESeq2.

For miRNA seed analysis, the longest 3' UTR isoform for each gene was considered. UTR annotations were obtained from the WS263 GTF annotation from Wormbase. Cytoscape was used to generate the network analysis graphs (25).

ChIP-seq data mapping, peak calling, and normalization

HSF-1 and Pol II ChIP-seq data were obtained from Li et al., 2016 (GEO Accession Number GSE81523) (26). Sequencing reads were aligned to a non-repeat-masked version of the $C$. elegans $\mathrm{N} 2$ reference genome (ce11) using Bowtie2 with the command bowtie 2 -no-unal --very-sensitive (27). HSF-1 peaks present at $34^{\circ} \mathrm{C}$ and their summits were called from Bowtie2-aligned reads with the MACS2 command macs2 callpeak -g ce —-keep-dup auto -call-summits -q 1e-6 using combined biological replicates and the single input replicate available (28). To normalize ChIP-seq data for display purposes, Bowtie2-mapped reads from combined biological replicates were filtered for duplicates using macs2 filterdup -g ce --keep-dup auto, and the condition with more mapped reads after filtering was randomly sampled down using macs 2 randsample so the total number of reads considered were identical between conditions. Finally, pileup of filtered reads was performed using macs 2 pileup with the --extsize parameter set to the fragment lengths predicted by MACS2 during peak-calling steps.

\section{HSE identification and scanning}

Motifs enriched in 101-bp non-repeat-overlapping HSF-1 summit regions were identified using MEME with the command meme -mod zoops -dna -revcomp (29). The most 
significant motif identified in HSF-1 peak summits closely resembles the previouslyidentified HSE motif using the same dataset (26). To scan the $C$.

elegans (ce11) genome for HSE locations, MEME-derived output for HSEs was used in conjunction with FIMO and its default parameters, which reports identified HSEs with $p<1$ e-04. HSF-1-bound HSEs were defined as those with at least 14-bp overlap with 201-bp regions centered on HSF-1 summits (30).

\section{HSF-1 RNAi and overexpression}

RNAi was performed by feeding animals either empty vector or hsf-1(RNAi). RNAi experiments were performed as described in (31). The HSF-1 overexpression strain EQ87, hsf-1p::hsf-1::gfp + rol-6, was used for HSF-1 overexpression studies $(32,33)$.

\section{qRT-PCR}

qPCR was performed as described in (34) except that ama-1 was used as a reference gene and the Quant Studio machine was used for all experiments. Primer sequences are as follows:

ama-1

Forward: CACTGGAGTTGTCCCAATTCTTG

Reverse: TGGAACTCTGGAGTCACACC

dct-10

Forward: GTCACACAGCCAACGAATG

Reverse: GTCGGAACTGTACGGATCAT

Helitron1_CE

Forward: AATCGTCGTGCCAATACCTC

Reverse: GTGCTCACCGAGATGTCTGA

hsp-16.2

Forward: GCTCTGATGGAACGCCAATTTGC

Reverse: CTGTGAGACGTTGAGATTGATGGCAAAC

hsp-70

Forward: CCGCTCGTAATCCGGAGAATA CAG

Reverse: CAACCTCAACAACGGGCTTTCC

linc-7

Forward: ACCAAGCAGACCCACCCT

Reverse: GTTGATGACGAGACGAGTGTGAG

\section{Data availability}

The RNA-seq datasets generated in this study will be deposited in a public repository upon acceptance of the manuscript. Other data and reagents are available upon request.

\section{RESULTS}

Multiple classes of RNA genes respond to heat shock in C. elegans 
The transcriptional response of protein-coding genes (PCGs) to Heat Shock (HS) has been intensively studied $(1,2,35)$, yet the effect of this stress on non-coding RNA (ncRNA) expression has not been examined systematically. Here we used RNA sequencing to compare the levels of various types of RNA from last larval stage (L4) C. elegans maintained at $20^{\circ} \mathrm{C}$ (control, CTRL) or shifted to $35^{\circ} \mathrm{C}$ (heat shock, HS) for 4 (RNA-seq) or 6 hours (smRNA-seq). Along with the expected HS-induced changes in mRNA expression (Supplementary Table S1), this profiling also revealed specific RNA genes belonging to the miRNA, piRNA, lincRNA, ncRNA, pseudogene and repeat families that changed at least two-fold in response to heat stress (Figure 1A).

While our conditions elicited changes in protein-coding mRNA expression comparable to previously published studies of the transcriptional response to HS in C. elegans (36, 37 ), we noticed some peculiarities in the up-regulated gene set. Visual inspection of the sequencing reads mapped to the UCSC Genome Browser indicated several false positive gene calls present in the list of PCGs up-regulated by HS. For example, srt-42 was originally listed as the second most highly up-regulated gene in HS but reads associated with this gene did not conform to the expected splicing pattern and included sequences stretching upstream into the neighboring heat shock protein gene, $h s p-16.41$ (Figure 1B). Thus, the reads covering sit-42 likely emanate from transcripts that failed to be properly terminated from the highly HS-induced $h s p-16.41$ gene. These types of aberrant transcripts, known as DoGs for Downstream of Gene containing transcripts, have been previously detected in various stress conditions $(38,39)$. While a functional role for DoGs is yet to be demonstrated, incomplete transcriptional termination of a highly induced upstream gene can provide RNA-seq reads that falsely assign a downstream gene on the same strand as also up-regulated $(38,39)$. Since these false positives are unlikely to maintain coding potential, we filtered them from our original list of HS up-regulated PCGs by removing 49 genes with reads in the upstream Intergenic Junction that were 2-fold higher in $\mathrm{HS}$ and that had a ratio of intronic to exonic reads of 0.4 or greater (Figure 1B and Supplementary Table S1).

Another source of mistakenly called up-regulated PCGs was the intronic residence of tRNAs and snoRNAs that apparently failed to be properly terminated in HS (Figure 1B). These longer versions of tRNAs and snoRNAs enabled them to be detected in standard RNA-seq, whereas the normally shorter forms are not captured in this procedure. By filtering out genes with an intron to exon ratio (IR) greater than 1 due to accumulation of an intronic ncRNA in HS, we removed another 14 PCGs from the up-regulated list (Figure 1B and Supplementary Table S1). Finally, PCGs that overlapped a repetitive element that was likely responsible for the increased sequencing reads in HS were also filtered out (Figure 1B and Supplementary Table S1). The bioinformatic steps used for filtering are detailed in the Materials and Methods and available online (https://github.com/wschrein).

With a higher confidence set of PCGs differentially regulated by $\mathrm{HS}$, we confirmed that our conditions elicited changes in mRNA expression comparable to previously published studies of the transcriptional response to HS in C. elegans $(36,37,40)$. The extensive changes in PCG expression induced by $\mathrm{HS}$ aligned with molecular pathways 
expected to be regulated by this stress. DAVID Gene Ontology (GO) analyses of genes up-regulated by HS indicated strong enrichment for those associated with cuticle maintenance, heat shock proteins, and enzymatic factors (Figure 1C; Supplementary Table S1) $(41,42)$. Genes in these categories have well-established roles in stress protection and have been previously found to be up-regulated in HS (Figure 1C; Supplementary Table S1) $(26,36,37)$. Distinct functional annotations, largely involved in nucleic acid binding, were enriched in the genes down-regulated by heat shock (Figure 1C; Supplementary Table S1). Decreased expression of genes associated with DNA replication is consistent with halted growth and cell division triggered by heat stress (1).

The up- and down-regulated gene-sets also pointed to distinct tissue responses to HS, consistent with cell non-autonomous regulation of the HSR in C. elegans $(43,44)$. Using Tissue Enrichment Analysis (TEA), we observed that up-regulated genes were associated with neuronal and epithelial cells (Figure 1D) (45). In particular, enrichment in thermosensory neurons may reflect the role of these neurons in activating the HSR in a cell non-autonomous manner (Figure 1D) (43). Down-regulated PCGs were associated with reproductive tissues, which likely corresponds to the delay of development in stress conditions.

Having confirmed that the heat shock conditions used in these studies elicited the expected changes in protein-coding gene expression, we next examined the effect of HS on major classes of ncRNA genes. The finding that particular members of each ncRNA class were up- or down-regulated in response to HS suggests that the Heat Shock Response controls the transcription or stability of specific ncRNAs.

\section{Specific miRNAs respond to thermal stress}

Of the 205 miRNAs we detected by small RNA sequencing, 8 were up-regulated at least 2-fold in C. elegans subjected to HS compared to CTRL conditions (Figure 2A and B; Supplementary Table S2). Consistent with a previous study, miR-239b was one of the most highly up-regulated miRNAs following HS (Figure 2A-B) (11). The largest fold change was observed for miR-4936, which increased by over 200-fold in HS (Figure 2AC). This miRNA was virtually undetectable at the CTRL temperature but accumulated to high levels in heat shock (Figure 2C). Furthermore, the predominant isoform we detected for miR-4936 in HS ( ${ }^{5}$ AUUGCUUUGUGGCUUUGCUGGUAAC ${ }^{3}$ ) differs from the reference sequence listed at miRBase ( ${ }^{\prime}$ UGCUUUGUGGCUUUGCUGGUA ${ }^{3}$ ) (46). This difference in 5'-end sequence is expected to affect target recognition, which is largely driven through initial pairing of miRNA nucleotides 2-8 (seed sequence) (12). Since target mRNA degradation is often the outcome of miRNA regulation, we searched the set of down-regulated PCGs for complementary sites in their 3'UTRs to the seed sequence (nt 2-8) of up-regulated miRNAs (Supplementary Table S2). The predicted targets of these miRNAs reflect GO terms associated with genes whose levels decrease in heat stress, such as 3'UTR binding (Figure 2B). Furthermore, some of these downregulated genes are potential targets of multiple up-regulated miRNAs, suggesting cooperativity (Figure 2D). These observations are consistent with a role for the upregulated miRNAs in contributing to the repression of some PCGs in HS. 
Whereas 52 miRNAs were found to be down-regulated in HS, most of these were the lowly expressed passenger strands of the initially processed miRNA duplex (Supplementary Table S2). The levels of only 15 guide strand miRNAs were reduced more than 2-fold after heat shock (Figure 2A-B; Supplementary Table S2). Target predictions for the 11 down-regulated miRNAs that were among the 100 most abundant miRNAs revealed top enriched GO terms associated with molting and movement (Figure 2B). Twenty-one of these target genes have the potential for recognition by 3 or more miRNAs down-regulated by HS, raising the possibility that increased expression of these genes is facilitated by alleviated miRNA repression (Figure 2E). Interestingly, the Period protein homolog lin-42 is strongly up-regulated in HS and a predicted target of four different miRNAs with reduced expression in HS (Figure 2E; Supplementary Table S2). This gene regulates molting cycles and acts as a general transcriptional repressor of miRNA genes (47-50). Thus, increased expression of lin-42 in HS could contribute to transcriptional repression of many miRNA genes. This would be consistent with the decreased levels of passenger strand miRNAs, which are more sensitive to changes in transcription than the stable guide strands.

In addition to miRNAs, we also identified 11,979 different piRNAs in our small RNA sequencing data sets. Of these, 15 increased and 63 decreased by at least 2-fold in HS compared to control conditions (Supplementary Table S2). Using the piRTarBase piRNA target prediction tool (51), we found that very few differentially regulated PCGs have potential for regulation by these piRNAs (Supplementary Table S2). Thus, the limited differences in piRNA levels are unlikely to contribute much to the extensive changes in PCG expression observed in HS.

\section{Specific long non-coding RNAs accumulate in HS}

In contrast to miRNAs, where similar numbers of the more abundant species were upand down-regulated in HS, longer ncRNAs primarily increased in levels in response to heat stress. Approximately 150 genes are annotated as long intergenic non-coding RNAs (lincRNAs) in $C$. elegans (52). While functional roles for the lincRNAs are largely unknown, the lincRNA genes tts-1 and rncs-1 have been associated with longevity and stress pathways, respectively $(14,15)$. Through our RNA profiling, we found that seven relatively abundant lincRNAs increased and only one lincRNA decreased by more than two-fold in HS compared to CTRL conditions (Figure 3A; Supplementary Table S3). Interestingly, tts-1 was among the highly up-regulated lincRNAs, suggesting that its role in longevity may be linked to a stress pathway (14). At least one of the lincRNAs, linc-7, appears to be rapidly up-regulated by HS (Figure 3B). Interestingly, this lincRNA contains five sites with complementarity to the seed region of the rapidly up-regulated miRNA, miR-239b-5p. Additionally, linc-82 contains eight seed matches for miR-239a$3 p$ and miR-230-3p, both of which accumulate in HS (Figure 2A-B; Supplementary Table S2). These potential lincRNA-miRNA interactions may regulate the stability, availability or function of these specific RNAs during HS.

In addition to lincRNAs, over 4600 ncRNAs that do not belong to a previously characterized class of non-coding RNA are annotated in the C. elegans genome (22). 
Of these, 71 increased and 1 decreased at least two-fold in response to HS (Figure 3C; Supplementary Table S3). We also detected expression for 1455 pseudogenes and found that 94 were up- and 23 were down-regulated in HS (Figure 3D; Supplementary Table S4). The relatively large number of pseudogene RNAs that accumulated to high levels in HS suggests that elevated temperatures disrupt the surveillance pathways that normally suppress pseudogene expression.

\section{Repetitive element-derived RNAs accumulate in heat shock}

By re-mapping our RNA-sequencing data to a list of consensus $C$. elegans repetitive elements obtained from repbase (24), we identified reads for 165 types of repetitive elements in the $C$. elegans genome. Similar numbers of repeats $(\sim 20)$ were found to increase or decrease in HS; however, the magnitude of change was greatly amplified in the up-regulated set (Figure 4A-B; Supplementary Table S4). The apparent strong upregulation of CERP16 is likely due, at least in part, to the position of this element immediately downstream of $h s p-16.2$ and $h s p-16.41$. The DoGs produced by each of these highly induced HSP genes are likely a predominant source of reads for this repeat element.

In contrast, at least some members of the rolling circle DNA transposons known as Helitrons appeared to be strongly induced independently of neighboring PCGs (Figure 4B and see below). RNA from Helitron1_CE rapidly accumulated to high levels upon HS (Figure 4C-D). Our reanalysis of previously published C. elegans HS RNA-Seq data also confirmed fast induction of Helitron repetitive element RNA (36).

\section{HSF-1 controls the expression of specific ncRNAs}

While defective small RNA silencing pathways may explain some of the increased repeat RNA expression during HS (53), the rapid and massive accumulation of Helitron RNAs suggests that transcriptional induction is also involved. We noted that Helitron1_CE exhibited a heat shock response comparable to that of canonical HSinduced genes, such as $h s p-16.42$, with barely detectable RNA levels that quickly rise upon HS (Figure 4C). Thus, we predicted that, like HSP genes, Helitrons could be direct transcriptional targets of HSF-1. To investigate this possibility, we remapped $C$. elegans HSF-1 and Pol II ChIP-Seq data from Li and colleagues to include repeat and other ncRNA genomic loci (26). Similar to the promoter region for $h s p-16.2$ and $h s p-16.41$, we found evidence for HSF-1 and Pol II association with Helitron1_CE, and other Helitron family members, in response to HS (Figure 5A; Supplementary Tables S1 and S4). Furthermore, the HSF-1 peaks overlapped with multiple copies of Heat Shock Response Elements (HSEs) present in the Helitron genes (Figure 5A; Supplementary Table S4).

The interaction of HSF-1 with Helitron regions suggests that HSF-1 could directly contribute to the up-regulation of Helitron RNA in HS. Using conditions that decrease (hsf-1(RNAi)) or increase (transgenic overexpression; $h s f-1(\mathrm{OEX})$ ) the levels of HSF-1, we observed the expected opposite effects on the expression of $h s p-16.2$, an established HSF-1 target, in response to HS (Figure 5B). Likewise, the induction of Helitron1_CE expression by HS was significantly reduced in $h s f-1$ (RNAi) and enhanced 
in $h s f-1(\mathrm{OEX})$ conditions (Figure 5B). Given the abundance of HSEs present in some Helitrons and the sensitivity of Helitron1_CE to HSF-1 levels, up-regulation of these repeat genes during $\mathrm{HS}$ may be largely regulated at the transcriptional level.

To explore the possibility that other ncRNAs may be part of the direct HSF-1 transcriptional program in HS, we analyzed their putative promoter regions $(1 \mathrm{~kb}$ upstream of the annotated start site) for HSEs and HSF-1 peaks in the ChIP-seq data from Li et al., 2016 (26). Of the up-regulated miRNAs, only the miR-239 locus fit these criteria (Supplementary Table S2). This region is situated between miR-239b and miR239a, which are transcribed in opposite directions. The single HSE and greater level of HSF-1 ChIP-seq reads are closer to the start of miR-239b, but enhanced Pol II occupancy in HS is observed over both miRNAs, consistent with their mutual upregulation in HS (Figures 2A-B and 5A; Supplementary Table S2). Furthermore, we found that reducing or increasing HSF-1 levels resulted in lower or higher miR-239b levels, respectively, in response to HS (Figure 5B). For the up-regulated longer ncRNA genes, HSEs and evidence of HSF-1 binding in HS were detected in the promoter regions of 0 lincRNAs, 11 ncRNAs, and 5 pseudogenes (Supplementary Table S4). Consistent with the DAF-16/FOXO-controlled tumor gene, dct-10, being one of the most robustly induced pseudogenes, we found that its promoter contains multiple HSEs and its expression in HS is regulated by $h s f-1$ (Figure 5; Supplementary Table S4). Altogether, these findings point to an expanded role for HSF-1 in directing the transcription of specific ncRNA genes as part of the heat shock response in $C$. elegans.

\section{DISCUSSION}

Here we surveyed the response of multiple classes of ncRNA, as well as protein-coding, genes to an episode of HS in $C$. elegans. Our analysis shows that, of the currently annotated genes, HS induced at least a two-fold change in the expression of approximately 9\% PCGs, 5\% miRNAs, 0.5\% piRNAs, 6\% lincRNAs, $0.1 \%$ ncRNAs, $6 \%$ pseudogenes and $24 \%$ of the repeat families. Furthermore, some of the most upregulated ncRNAs, such as miR-4936, were barely detectable under control temperature conditions, demonstrating that often ignored, lowly expressed genes should be reconsidered in different contexts. Our finding that several ncRNA genes parallel canonical HSR genes, such as HSPS, in their dependence on HSF-1 for rapid induction suggests that regulatory RNAs may also have important roles in mitigating the damage caused by excessive heat.

\footnotetext{
Aberrant 3'-extended transcripts accumulate in heat shock

During our analysis of differentially regulated PCGs in control versus HS conditions, we noticed a previously described phenomenon known as DoGs for Downstream of Genes (38-40). DoGs result from transcriptional readthrough, leading to mRNAs with long 3' extensions that include normally intergenic sequence $(38,39)$. Sometimes DoGs read into adjacent downstream PCGs, which can result in aberrant gene calls (38). Thus, the reads assigned to the downstream gene actually belong to transcripts that are chimeric with the upstream gene and are unlikely to retain coding potential. Such false positives
} 
are particularly problematic for compact genomes where closely spaced genes reside in the same orientation. To deal with this issue in our set of PCGs up-regulated in HS, we applied two filters to remove candidates likely emanating from DoGs. We found it necessary to combine the criteria of increased intergenic junction and intron reads because $C$. elegans 5'UTRs are incompletely annotated and splicing is generally less efficient in HS conditions $(7,54)$ This filtering pipeline may be useful for analyzing other C. elegans RNA-seq datasets, since DoGs seem to be generated by a variety of stress conditions (38-40).

The reason for transcriptional readthrough and DoGs generation during stress is currently unclear (38). Since transcriptional termination of most PCGs by RNA Pol II involves the cleavage and polyadenylation machinery, it is conceivable that this process is generally less efficient at elevated temperatures (55). The termination step can also be influenced by chromatin architecture and structural context of the poly $(A)$ signal, which may be sensitive to temperature changes (55). Furthermore, transcriptional termination efficiency by RNA Pol III also seems reduced in HS. We detected 3'extended tRNA and snoRNA transcripts in the HS, but not control, RNA samples; whereas the mature forms of these Pol III RNAs are too short for capture in standard RNA-Seq library preparations. Since tRNA and snoRNA genes are commonly embedded in introns of PCGs in C. elegans, the extended forms observed in HS sometimes overlapped exons. We were able to filter out these falsely called upregulated PCGs by removing genes with intron retention scores greater than 1 . While the cause of 3'-extended Pol II and III transcripts in heat shocked C. elegans is yet to be determined, an awareness that these aberrant transcripts can accumulate is important for understanding changes in coding potential induced by stress.

\section{Specific ncRNAs from multiple classes respond to Heat Shock}

Although the majority of individual $C$. elegans miRNA genes are apparently dispensable under laboratory growth conditions, there is accumulating evidence that specific miRNAs play integral roles in a variety of stress response pathways (56-58). Previous studies found that loss of miR-71, miR-246, miR-80, or the miR-229, -64, -66 cluster resulted in increased sensitivity to heat stress $(11,13)$. We observed that miR-71 is upregulated 1.4-fold in HS (Supplementary Table S2), which would be consistent with a survival role for this miRNA in elevated temperatures. Conversely, we detected an over 5 -fold decrease in miR-246 levels in response to HS, which seems contrary to the profound sensitivity of miR-246 mutants to HS (13). Another counterintuitive change in expression was observed for miR-239a/b. We, and others, detected substantial increases in the levels of these related miRNAs in response to HS (11). Additionally, we present evidence that these miRNAs are transcriptionally induced by HSF-1. Yet, the miR-239a/b(nDf62) strain, which lacks both miRNAs, was previously reported to have an enhanced survival phenotype when subjected to HS $(11,13)$. The identification of direct targets of the differentially regulated miRNAs will be necessary to understand how their altered expression during HS relates to their functional roles.

Most of the miRNAs we found to be up- or down-regulated by HS have not yet been ascribed biological functions. Of the miRNAs that increase in HS, previous reports have 
documented that miR-247 promotes survival upon exposure to graphene oxide and miR-235 regulates developmental arrest in response to starvation $(59,60)$. Our observation that these miRNAs increase in HS suggests that they may have roles in multiple stress response pathways. Down-regulation of miR-34 in response to our HS conditions was surprising given its reported roles in promoting dauer survival in response to food deprivation and ensuring normal development in animals subjected to rapid temperature fluctuations $(61,62)$. However, miR-34 mutants have also been found to exhibit increased and decreased radiosensitivity in the soma and germline, respectively, suggesting that the function of this miRNA is highly context dependent (63).

By far, the most changed miRNA was miR-4936, which increased over 100-fold in HS. Although the dramatic fold change is linked to the virtually undetectable levels in control conditions, this miRNA did accumulate to appreciable levels, as validated by Northern blot. Curiously, this miRNA exhibited more heterogeneity in its mature forms than most other miRNAs. In fact, the predominant species detected in our HS studies differs from the sequence currently present in miRbase (46). While the miR-4936 sequence resides in a predicted hairpin that resembles other miRNA precursors, a corresponding passenger strand has not yet been identified that would be indicative of canonical Dicer processing. Regardless of these peculiarities, it is evident that HS induces massive upregulation of this RNA, making it a contender for a role in the HSR.

Unlike the comparable numbers of up- and down-regulated miRNAs, members of the longer ncRNA classes predominately increased in HS. Considering that very few functions have been assigned to any $C$. elegans long ncRNAs, the ones that increase in HS are good candidates for potential roles in this stress condition. The lincRNA tts-1 (transcribed telomeric sequence) has previously been shown to be important for the extended lifespan of animals with reduced insulin signaling (daf-2 mutants) or mitochondrial activity (clk-1 mutants) (14). As the levels of tts-1 are increased in long lived daf-2 mutants and in animals grown in the presence of bacterial pathogens, the over 10-fold increase in tts-1 RNA induced by HS could reflect a general response of this lincRNA to stress $(14,64)$.

Some mammalian long ncRNAs, including pseudogene RNAs, have been assigned roles as competitive endogenous RNAs (ceRNAs) (65). In this role, long ncRNAs have the potential to sequester and sometimes destabilize small RNAs, such as miRNAs, through base-pairing interactions. A caveat to broad regulation of miRNA availability by ceRNAs, is the typically much lower abundance of lincRNAs and pseudogene RNAs (66). Thus, dramatic up-regulation of these types of RNAs, as we observed for specific lincRNAs, ncRNAs and pseudogenes during HS, could boost their regulatory potential. Notably, the up-regulated linc-7 contains 5 sites that support seed pairing to miR-239a$5 p$ and miR-239b-5p. The linc-82 contains repeating elements that can pair with the other half of miR-239a, referred to as the $-3 p$ or passenger strand, as well as with miR230-3p. Considering that these lincRNAs and their potential miRNA partners are all upregulated in HS, mutual RNA stabilization could be an outcome of these interactions. 
There is precedent for repeat RNAs being expressed at higher levels in response to increased temperature (67-70). In C. elegans, even a mild temperature elevation can trigger aberrant expression of repetitive loci. Growth at $25^{\circ} \mathrm{C}$, instead of the standard condition of $20^{\circ} \mathrm{C}$, resulted in increased levels of RNA from some transposons (70). Given the importance of silencing transposons and maintaining genome integrity, it is not surprising that multiple small RNA and chromatin remodeling pathways act to prevent the mobilization of repetitive elements under normal and stress conditions $(70$, 71). Nonetheless, the up-regulation of RNAs from multiple repeat families during HS indicates that these silencing pathways become compromised in harsh environments. Interestingly, the HS-induced change in repeat RNA expression can be inherited and last for multiple generations in the absence of the original heat stress (70). It has been proposed that certain repeat RNAs or transposon mobilization could be advantageous during stress (72). For example, SINE RNAs in human and mouse cells are induced by HS and act as transcriptional repressors by binding directly to RNA Pol II $(73,74)$. Additionally, changes in genomic arrangement caused by stress induced expression of transposons could foster beneficial changes in the progeny of stressed parents. Evidence for this idea was recently documented in Schizosaccharomyces pombe, where certain forms of stress induced the mobility of transposable elements and the new insertions were linked to enhanced adaptation to the assault (75). As elevated temperatures have been associated with increased genetic mobility in C. elegans (76), it is possible that relaxed silencing of transposons during HS could sometimes provide an advantageous genetic change in the progeny of stressed animals.

\section{HSF-1 regulates the expression of diverse ncRNAs}

Activation of the transcription factor HSF-1 in response to HS, and a variety of other environmental perturbations, is essential for driving the expression of factors needed to survive the stress (35). While numerous studies, including our own, have observed the up-regulation of hundreds of PCGs in response to HS (35), recent work in mammalian cells and budding yeast has shown that HSF1/Hsf1 is directly responsible for the transcriptional induction of only a small subset of these genes, which mostly encode molecular chaperones $(4,5)$. Although it is yet to be established if HSF-1 has a similar limited set of direct essential targets in intact multicellular organisms, it is evident that $\mathrm{HS}$ induces widespread changes in HSF-1 binding across the genome in C. elegans (26). Here we show that some of these HSF-1-bound loci are associated with ncRNA genes. We identified 2 miRNA, 11 ncRNA, 5 pseudogene, and 5 repeat family genes that were up-regulated in HS and contained HSEs centered within HSF-1 ChIP-seq peaks. Furthermore, we confirmed that the up-regulation of miR-239b, Helitron1_CE, and pseudogene dct-10, induced by HS was sensitive to HSF-1 levels. A previous study, found that six C. elegans miRNAs were dependent on HSF-1 for increased expression in HS (10). Thus, the network of miRNAs, and other ncRNAs, controlled by HSF-1, both directly and indirectly, expands the repertoire of genes regulated by this transcription factor during the HSR. Furthermore, the dramatic, and in some cases rapid, accumulation of specific ncRNAs in response to heat stress in C. elegans suggests that functions for some regulatory RNAs may only surface when needed for organismal survival in the natural world. 


\section{FUNDING}

This work was supported by the National Institutes of Health [R35 GM127012 to A.E.P, T32 GM007240 to W.P.S and D.C.P., F32 GM112426 to J.S.C.], the National Cancer Institute [T32 CA009523 to J.M.G.], and the Sigrid Jusélius Foundation and the Finnish Cultural Foundation to A.P.A.

\section{ACKNOWLEDGMENTS}

We thank Dr. Cindy M. Voisine and members of the Pasquinelli Lab for helpful discussions and critical reading of the manuscript. 


\section{REFERENCES}

1. Richter,K., Haslbeck,M. and Buchner,J. (2010) The heat shock response: life on the verge of death. Mol. Cell, 40, 253-66.

2. Åkerfelt,M., Morimoto,R.I. and Sistonen,L. (2010) Heat shock factors: integrators of cell stress, development and lifespan. Nat. Rev. Mol. Cell Biol., 11, 545-555.

3. Duarte,F.M., Fuda,N.J., Mahat,D.B., Core,L.J., Guertin,M.J. and Lis,J.T. (2016) Transcription factors GAF and HSF act at distinct regulatory steps to modulate stress-induced gene activation. Genes Dev., 30, 1731-46.

4. Mahat,D.B., Salamanca,H.H., Duarte,F.M., Danko,C.G. and Lis,J.T. (2016) Mammalian Heat Shock Response and Mechanisms Underlying Its Genome-wide Transcriptional Regulation. Mol. Cell, 62, 63-78.

5. Solís,E.J., Pandey,J.P., Zheng,X., Jin,D.X., Gupta,P.B., Airoldi,E.M., Pincus,D. and Denic,V. (2016) Defining the Essential Function of Yeast Hsf1 Reveals a Compact Transcriptional Program for Maintaining Eukaryotic Proteostasis. Mol. Cell, 63, 6071.

6. Leung,A.K.L. and Sharp,P.A. (2010) MicroRNA Functions in Stress Responses. Mol. Cell, 40, 205-215.

7. Shalgi,R., Hurt,J.A., Lindquist,S. and Burge,C.B. (2014) Widespread inhibition of posttranscriptional splicing shapes the cellular transcriptome following heat shock. Cell Rep., 7, 1362-1370.

8. Zhou,J., Wan,J., Gao,X., Zhang,X., Jaffrey,S.R. and Qian,S.B. (2015) Dynamic m6 A mRNA methylation directs translational control of heat shock response. Nature, 526, 591-594.

9. Di Giammartino,D.C., Shi,Y. and Manley,J.L. (2013) PARP1 Represses PAP and Inhibits Polyadenylation during Heat Shock. Mol. Cell, 49, 7-17.

10. Brunquell,J., Snyder,A., Cheng,F. and Westerheide,S.D. (2017) HSF-1 is a regulator of miRNA expression in Caenorhabditis elegans. PLoS One, 12.

11. Nehammer,C., Podolska,A., Mackowiak,S.D., Kagias,K. and Pocock,R. (2015) Specific microRNAs Regulate Heat Stress Responses in Caenorhabditis elegans. Sci. Rep., 5, 8866.

12. Bartel,D.P. (2018) Metazoan MicroRNAs. Cell, 173, 20-51.

13. De Lencastre,A., Pincus,Z., Zhou,K., Kato,M., Lee,S.S. and Slack,F.J. (2010) MicroRNAs both promote and antagonize longevity in C. elegans. Curr. Biol., 20, 2159-2168.

14. Essers,P.B., Nonnekens,J., Goos,Y.J., Betist,M.C., Viester,M.D., Mossink,B., Lansu,N., Korswagen,H.C., Jelier,R., Brenkman,A.B., et al. (2015) A Long Noncoding RNA on the Ribosome Is Required for Lifespan Extension. Cell Rep., 10, 339-345.

15. Hellwig,S. and Bass,B.L. (2008) A starvation-induced noncoding RNA modulates expression of Dicer-regulated genes. Proc. Natl. Acad. Sci. U. S. A., 105, 12897902.

16. Horváth,V., Merenciano,M. and González,J. (2017) Revisiting the Relationship between Transposable Elements and the Eukaryotic Stress Response. Trends Genet., 33, 832-841.

17. Wood,W. (1988) The Nematode Caenorhabditis elegans Cold Spring Harbor Laboratory, Cold Spring Harbor, New York, USA. 
18. Dobin,A., Davis,C.A., Schlesinger,F., Drenkow,J., Zaleski,C., Jha,S., Batut,P., Chaisson,M. and Gingeras,T.R. (2013) STAR: Ultrafast universal RNA-seq aligner. Bioinformatics, 29, 15-21.

19. Li,H., Handsaker,B., Wysoker,A., Fennell,T., Ruan,J., Homer,N., Marth,G., Abecasis, G. and Durbin,R. (2009) The Sequence Alignment/Map format and SAMtools. Bioinformatics, 25, 2078-2079.

20. Liao,Y., Smyth,G.K. and Shi,W. (2014) FeatureCounts: An efficient general purpose program for assigning sequence reads to genomic features. Bioinformatics, 30, 923-930.

21. Love,M.I., Huber,W. and Anders,S. (2014) Moderated estimation of fold change and dispersion for RNA-seq data with DESeq2. Genome Biol., 15, 550.

22. Lee,R.Y.N., Howe,K.L., Harris,T.W., Arnaboldi,V., Cain,S., Chan,J., Chen,W.J., Davis,P., Gao,S., Grove,C., et al. (2018) WormBase 2017: Molting into a new stage. Nucleic Acids Res., 46, D869-D874.

23. Quinlan,A.R. and Hall,I.M. (2010) BEDTools: A flexible suite of utilities for comparing genomic features. Bioinformatics, 26, 841-842.

24. Bao,W., Kojima,K.K. and Kohany,O. (2015) Repbase Update, a database of repetitive elements in eukaryotic genomes. Mob. DNA, 6, 11.

25. Shannon,P., Markiel,A., Ozier,O., Baliga,N.S., Wang,J.T., Ramage,D., Amin,N., Schwikowski,B. and Ideker,T. (2003) Cytoscape: a software environment for integrated models of biomolecular interaction networks. Genome Res., 13, 2498504.

26. Li,J., Chauve,L., Phelps,G., Brielmann,R.M. and Morimoto,R.I. (2016) E2F coregulates an essential HSF developmental program that is distinct from the heatshock response. Genes Dev., 30, 2062-2075.

27. Langmead,B. and Salzberg,S.L. (2012) Fast gapped-read alignment with Bowtie 2. Nat. Methods, 9, 357-9.

28. Zhang,Y., Liu,T., Meyer,C.A., Eeckhoute,J., Johnson,D.S., Bernstein,B.E., Nussbaum,C., Myers,R.M., Brown,M., Li,W., et al. (2008) Model-based Analysis of ChIP-Seq (MACS). Genome Biol., 9, R137.

29. Bailey,T.L., Boden,M., Buske,F.A., Frith,M., Grant,C.E., Clementi,L., Ren,J., Li,W.W. and Noble,W.S. (2009) MEME Suite: Tools for motif discovery and searching. Nucleic Acids Res., 37.

30. Grant,C.E., Bailey,T.L. and Noble,W.S. (2011) FIMO: scanning for occurrences of a given motif. Bioinformatics, 27, 1017-1018.

31. Ahringer,J. (2006) Reverse genetics. WormBook, 10.1895/wormbook.1.47.1.

32. Chiang,W.-C., Ching,T.-T., Lee,H.C., Mousigian,C. and Hsu,A.-L. (2012) HSF-1 Regulators DDL-1/2 Link Insulin-like Signaling to Heat-Shock Responses and Modulation of Longevity. Cell, 148, 322-334.

33. Kumsta,C., Chang,J.T., Schmalz,J. and Hansen,M. (2017) Hormetic heat stress and HSF-1 induce autophagy to improve survival and proteostasis in C. elegans. Nat. Commun., 8, 14337.

34. Aalto,A.P., Nicastro,I.A., Broughton,J.P., Chipman,L.B., Schreiner,W.P., Chen,J.S. and Pasquinelli,A.E. (2018) Opposing roles of microRNA Argonautes during Caenorhabditis elegans aging. PLoS Genet., 14, e1007379.

35. Vihervaara,A., Duarte,F.M. and Lis,J.T. (2018) Molecular mechanisms driving 
transcriptional stress responses. Nat. Rev. Genet., 19, 385-397.

36. Brunquell,J., Morris,S., Lu,Y., Cheng,F. and Westerheide,S.D. (2016) The genomewide role of HSF-1 in the regulation of gene expression in Caenorhabditis elegans. BMC Genomics, 17, 559.

37. Jovic,K., Sterken,M.G., Grilli,J., Bevers,R.P.J., Rodriguez,M., Riksen,J.A.G., Allesina,S., Kammenga,J.E. and Snoek,L.B. (2017) Temporal dynamics of gene expression in heat-stressed Caenorhabditis elegans. PLoS One, 12, e0189445.

38. Vilborg,A., Sabath,N., Wiesel,Y., Nathans,J., Levy-Adam,F., Yario,T.A., Steitz,J.A. and Shalgi,R. (2017) Comparative analysis reveals genomic features of stressinduced transcriptional readthrough. Proc. Natl. Acad. Sci., 114, E8362-E8371.

39. Vilborg,A., Passarelli,M.C., Yario,T.A., Tycowski,K.T. and Steitz,J.A. (2015) Widespread Inducible Transcription Downstream of Human Genes. Mol. Cell, 59, 449-461.

40. Melnick,M., Gonzales,P., Cabral,J., Allen,M.A., Dowell,R.D. and Link,C.D. (2019) Heat shock in $C$. elegans induces downstream of gene transcription and accumulation of double-stranded RNA. PLoS One, 14, e0206715.

41. Huang,D.W., Sherman,B.T. and Lempicki,R.A. (2009) Systematic and integrative analysis of large gene lists using DAVID bioinformatics resources. Nat. Protoc., 4 , 44-57.

42. Huang,D.W., Sherman,B.T. and Lempicki,R.A. (2009) Bioinformatics enrichment tools: Paths toward the comprehensive functional analysis of large gene lists. Nucleic Acids Res., 37, 1-13.

43. Prahlad,V., Cornelius,T. and Morimoto,R.I. (2008) Regulation of the cellular heat shock response in Caenorhabditis elegans by thermosensory neurons. Science (80-. )., 320, 811-814.

44. O'Brien,D. and van Oosten-Hawle,P. (2016) Regulation of cell-non-autonomous proteostasis in metazoans. Essays Biochem., 60, 133-142.

45. Angeles-Albores,D., N. Lee,R.Y., Chan,J. and Sternberg,P.W. (2016) Tissue enrichment analysis for C. elegans genomics. BMC Bioinformatics, 17, 366.

46. Kozomara,A., Birgaoanu,M. and Griffiths-Jones,S. (2019) MiRBase: From microRNA sequences to function. Nucleic Acids Res., 47, D155-D162.

47. McCulloch,K.A. and Rougvie,A.E. (2014) Caenorhabditis elegans period homolog lin-42 regulates the timing of heterochronic miRNA expression. Proc. Natl. Acad. Sci., 111, 15450-15455.

48. Monsalve,G.C., Van Buskirk,C. and Frand,A.R. (2011) LIN-42/PERIOD controls cyclical and developmental progression of C. elegans molts. Curr. Biol., 21, 20332045.

49. Van Wynsberghe,P.M., Finnegan,E.F., Stark,T., Angelus,E.P., Homan,K.E., Yeo,G.W. and Pasquinelli,A.E. (2014) The Period protein homolog LIN-42 negatively regulates microRNA biogenesis in C. elegans. Dev. Biol., 390, 126-135.

50. Perales,R., King,D.M., Aguirre-Chen,C. and Hammell,C.M. (2014) LIN-42, the Caenorhabditis elegans PERIOD homolog, Negatively Regulates MicroRNA Transcription. PLoS Genet., 10, e1004486.

51. Wu,W.S., Brown,J.S., Chen,T. Te, Chu,Y.H., Huang,W.C., Tu,S. and Lee,H.C. (2019) PiRTarBase: A database of piRNA targeting sites and their roles in gene regulation. Nucleic Acids Res., 47, D181-D187. 
52. Nam,J.-W. and Bartel,D.P. (2012) Long noncoding RNAs in C. elegans. Genome Res., 22, 2529-2540.

53. Weiser,N.E. and Kim,J.K. (2019) Multigenerational Regulation of the Caenorhabditis elegans Chromatin Landscape by Germline Small RNAs. Annu. Rev. Genet., 53, annurev-genet-112618-043505.

54. Yost,H.J. and Lindquist,S. (1986) RNA splicing is interrupted by heat shock and is rescued by heat shock protein synthesis. Cell, 45, 185-193.

55. Proudfoot,N.J. (2016) Transcriptional termination in mammals: Stopping the RNA polymerase II juggernaut. Science (80-. )., 352, aad9926-aad9926.

56. Ambros,V. and Ruvkun,G. (2018) Recent Molecular Genetic Explorations of Caenorhabditis elegans MicroRNAs. Genetics, 209, 651-673.

57. Miska,E.A., Alvarez-Saavedra,E., Abbott,A.L., Lau,N.C., Hellman,A.B., McGonagle,S.M., Bartel,D.P., Ambros, V.R. and Horvitz,H.R. (2007) Most Caenorhabditis elegans microRNAs are individually not essential for development or viability. PLoS Genet., 3, 2395-2403.

58. Alvarez-Saavedra,E. and Horvitz,H.R. (2010) Many Families of C. elegans MicroRNAs Are Not Essential for Development or Viability. Curr. Biol., 20, 367-373.

59. Liu,P., Shao,H., Ding,X., Yang,R., Rui,Q. and Wang,D. (2019) Dysregulation of Neuronal Gao Signaling by Graphene Oxide in Nematode Caenorhabditis elegans. Sci. Rep., 9, 6026.

60. Kasuga,H., Fukuyama,M., Kitazawa,A., Kontani,K. and Katada,T. (2013) The microRNA miR-235 couples blast-cell quiescence to the nutritional state. Nature, 497, 503-506.

61. Isik,M., Blackwell,T.K. and Berezikov,E. (2016) MicroRNA miR-34 provides robustness to environmental stress response via the DAF-16 network in C. elegans. Sci. Rep., 6, 36766.

62. Burke,S.L., Hammell,M. and Ambros,V. (2015) Robust Distal Tip Cell Pathfinding in the Face of Temperature Stress Is Ensured by Two Conserved microRNAS in Caenorhabditis elegans. Genetics, 200, 1201-18.

63. Kato,M., Chen,X., Inukai,S., Zhao,H. and Slack,F.J. (2011) Age-associated changes in expression of small, noncoding RNAs, including microRNAs, in C. elegans. RNA, 17, 1804-1820.

64. O’Rourke,D., Baban,D., Demidova,M., Mott,R. and Hodgkin,J. (2006) Genomic clusters, putative pathogen recognition molecules, and antimicrobial genes are induced by infection of C. elegans with M. nematophilum. Genome Res., 16, 10051016.

65. Grüll,M.P. and Massé,E. (2019) Mimicry, deception and competition: The life of competing endogenous RNAs. Wiley Interdiscip. Rev. RNA, 10, e1525.

66. Denzler,R., McGeary,S.E., Title,A.C., Agarwal,V., Bartel,D.P. and Stoffel,M. (2016) Impact of MicroRNA Levels, Target-Site Complementarity, and Cooperativity on Competing Endogenous RNA-Regulated Gene Expression. Mol. Cell, 64, 565-579.

67. Jolly,C., Metz,A., Govin,J., Vigneron,M., Turner,B.M., Khochbin,S. and Vourc'h,C. (2004) Stress-induced transcription of satellite III repeats. J. Cell Biol., 164, 25-33.

68. Goenka,A., Sengupta,S., Pandey,R., Parihar,R., Mohanta,G.C., Mukerji,M. and Ganesh,S. (2016) Human satellite-III non-coding RNAs modulate heat-shockinduced transcriptional repression. J. Cell Sci., 129, 3541-3552. 
69. Hogan,N.C., Slot,F., Traverse,K.L., Garbe,J.C., Bendena,W.G. and Pardue,M.L. (1995) Stability of tandem repeats in the Drosophila melanogaster Hsr-omega nuclear RNA. Genetics, 139, 1611-1621.

70. Klosin,A., Casas,E., Hidalgo-Carcedo,C., Vavouri,T. and Lehner,B. (2017) Transgenerational transmission of environmental information in C. elegans. Science (80-. )., 356, 320-323.

71. McMurchy,A.N., Stempor,P., Gaarenstroom,T., Wysolmerski,B., Dong,Y., Aussianikava,D., Appert,A., Huang,N., Kolasinska-Zwierz,P., Sapetschnig,A., et al. (2017) A team of heterochromatin factors collaborates with small RNA pathways to combat repetitive elements and germline stress. Elife, 6.

72. Horváth,V., Merenciano,M. and González,J. (2017) Revisiting the Relationship between Transposable Elements and the Eukaryotic Stress Response. Trends Genet., 33, 832-841.

73. Mariner,P.D., Walters,R.D., Espinoza,C.A., Drullinger,L.F., Wagner,S.D., Kugel,J.F. and Goodrich,J.A. (2008) Human Alu RNA Is a Modular Transacting Repressor of mRNA Transcription during Heat Shock. Mol. Cell, 29, 499-509.

74. Yakovchuk,P., Goodrich,J.A. and Kugel,J.F. (2009) B2 RNA and Alu RNA repress transcription by disrupting contacts between RNA polymerase II and promoter DNA within assembled complexes. Proc. Natl. Acad. Sci., 106, 5569-5574.

75. Esnault,C., Lee,M., Ham,C. and Levin,H.L. (2019) Transposable element insertions in fission yeast drive adaptation to environmental stress. Genome Res., 29, 85-95.

76. Matsuba,C., Ostrow,D.G., Salomon,M.P., Tolani,A. and Baer,C.F. (2012) Temperature, stress and spontaneous mutation in Caenorhabditis briggsae and Caenorhabditis elegans. Biol. Lett., 9, 20120334-20120334. 


\section{FIGURE LEGENDS}

Figure 1. Heat shock alters the expression of coding and non-coding RNAs.

(A) Small RNA-seq and stranded paired-end RNA-seq were used to analyze changes in RNA expression in C. elegans shifted from $20^{\circ} \mathrm{C}$ (control, CTRL) to $35^{\circ} \mathrm{C}$ (heat shock, HS) for 4 (RNA-seq) or 6 hours (smRNA-seq). The numbers of differentially expressed genes in each RNA category are indicated.

(B) Strategy used to filter out false positive up-regulated mRNAs. See methods for further details; codes used for filtering are available at https://github.com/wschrein (C) DAVID Functional Annotation Clustering of Genes up- and down-regulated in response to HS (41). Representative members of each cluster with an enrichment score $>2$ are shown. Size of dot corresponds to number of genes in each cluster.

(D) Tissue Enrichment Analysis (TEA) for genes up- and down-regulated in response to HS. TEA was performed using the Wormbase TEA tool (45). Abbreviations: PVD - Sensory neuron (polymodal nociceptive for mechanosensation and thermosensation), Hyp7 - entire syncytium of hyp7, Hyp6 - Cylindrical hypodermal syncytium in head, Psub1 - Embryonic founder cell, AB - Embryonic founder cell, Psub3 - Embryonic founder cell, EMS - Embryonic Cell, Z2 - Germ line precursor cell, Z3 Germ line precursor cell.

Figure 2. The expression of specific miRNAs is regulated by Heat Shock.

(A) Expression of miRNAs in CTRL versus HS detected by small RNA-Sequencing. Results represent the average of two independent biological replicates and miRNAs reproducibly up- (red) and down- (blue) regulated by $\geq 2$-fold are indicated.

(B) List of guide strand miRNAs within the top 100 expressed miRNAs that are reproducibly up- or down-regulated in response to HS. The final column shows the most highly enriched Molecular Function GO term of potential targets for each miRNA (see Supplementary Table S2). GO analysis was performed using DAVID (41).

(C) Analysis of miR-4936 expression in CTRL versus HS conditions by Northern blotting. 5S rRNA serves as a loading control.

(D-E) Network analysis of differentially regulated mRNAs targeted by at least 3 up- or down-regulated miRNAs. Cytoscape was used to draw networks (https://cytoscape.org) (25).

Figure 3. Heat shock alters the expression of long non-coding RNAs

(A) Expression of long intergenic non-coding RNAs (lincRNAs) in CTRL versus HS detected by stranded paired-end sequencing. Significantly up- (red) and down-regulated (blue) lincRNAs ( $\geq 2$-fold change with baseMean $\geq 50$ and padj $<0.05$ from 3 independent replicates) are indicated.

(B) qRT-PCR analysis of $h s p-70$ and linc-7 RNA levels after 15, 30 and 180 minutes of HS versus CTRL conditions. Mean fold changes and SEM from 3 independent replicates are shown. ${ }^{*} P<0.05$ (t-test, two-sided).

(C) Expression of ncRNAs in CTRL versus HS detected by stranded paired-end sequencing. Significantly up- (red) and down-regulated (blue) ncRNAs ( $\geq 2$-fold change with baseMean $\geq 50$ and padj $<0.05$ from 3 independent replicates) are indicated. 
(D) Expression of pseudogene RNAs in CTRL versus HS detected by stranded pairedend sequencing. Significantly up- (red) and down-regulated (blue) pseudogene RNAs ( $\geq$ 2 -fold change with baseMean $\geq 50$ and padj $<0.05$ from 3 independent replicates) are indicated.

Figure 4. Up-regulation of multiple repetitive element RNAs during heat shock (A) Expression of repetitive element RNAs in CTRL versus HS detected by stranded paired-end sequencing and mapped to a database of repetitive elements (24). Significantly up- (red) and down-regulated (blue) repeat RNAs ( $\geq 2$-fold change with baseMean $\geq 100$ and padj $<0.05$ from 3 independent replicates) are indicated.

(B) List of repetitive element RNAs up-regulated at least 10-fold in HS.

(C) Semi-quantitative RT-PCR detection of the indicated RNAs in CTRL and after 15 or 30 minutes of HS.

(D) Quantitative RT-PCR analysis of $h s p-70$ and Helitron1_CE RNA expression during a time course of heat shock. Mean fold changes and SEM from 3 independent replicates are shown. ${ }^{*} P<0.05,{ }^{* *} P<0.01,{ }^{* * *} P<0.001$ (t-test, two-sided).

Figure 5. NcRNAs are regulated by HSF-1 during heat shock.

(A) Genome browser screenshots of HSF-1 (yellow) and Pol II (blue) ChIP-seq data from control (CTRL) and heat shock (HS) conditions (data from (26)) for representative genes ( $h s p-16.2$ and $h s p-16.41$, mRNA; Helitron1_CE, repeat RNA, miR-239a and miR239b, miRNA; dct-10, pseudogene). Individual HSEs identified using FIMO $(P<1 \mathrm{e}-04)$ are indicated (30).

(B) Fold change in RNA levels of hsp16.2, Helitron1_CE, miR-239b, and dct-10 after 30 minutes of heat shock in animals subjected to empty vector or $h s f-1 \mathrm{RNAi}$, and Wildtype (WT) versus a strain overexpressing HSF-1 ( $h s f-1$ OEX) determined by Quantitative RTPCR analyses. The mean fold changes and SEM from 3 independent replicates are graphed. ${ }^{*} P<0.05,{ }^{* *} P<0.01$ (t-test, two-sided). 


\section{Figure 1}

A

Number of RNA species with $\mathbf{2}$ 2-fold change in expression after heat shock

UP DOWN ALL

\begin{tabular}{r|r|r|r|} 
piRNA & 15 & 15 & 437 \\
\hline
\end{tabular}

B

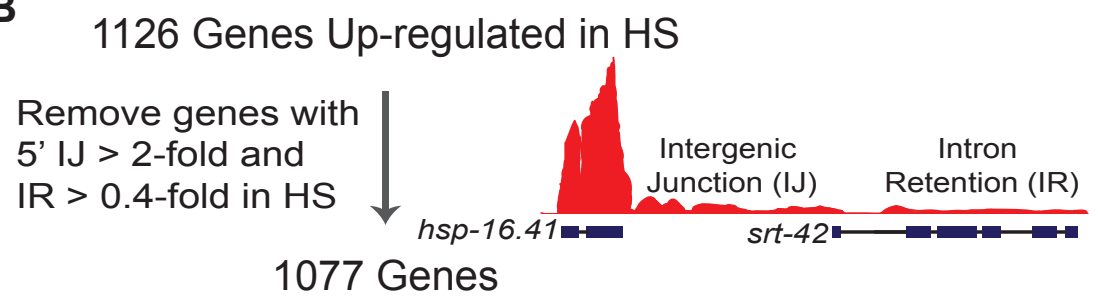

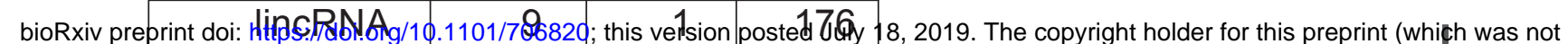

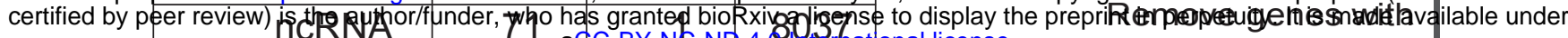

\begin{tabular}{|r|r|r|r|}
\hline pseudogene & 94 & 23 & 1922 \\
\hline repeat & 21 & 23 & 181 \\
\hline mRNA & 1059 & 801 & 20203 \\
\hline
\end{tabular}

$\mathrm{IR}>1.0$ in $\mathrm{HS}$

Y51H7BR.8

1063 Genes

Remove genes that overlap with $>50 \%$ of a Repeat

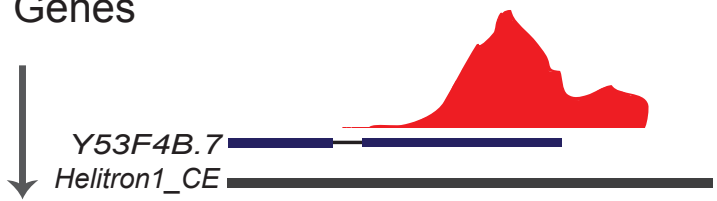

1059 Up-regulated

Protein-coding Genes

C
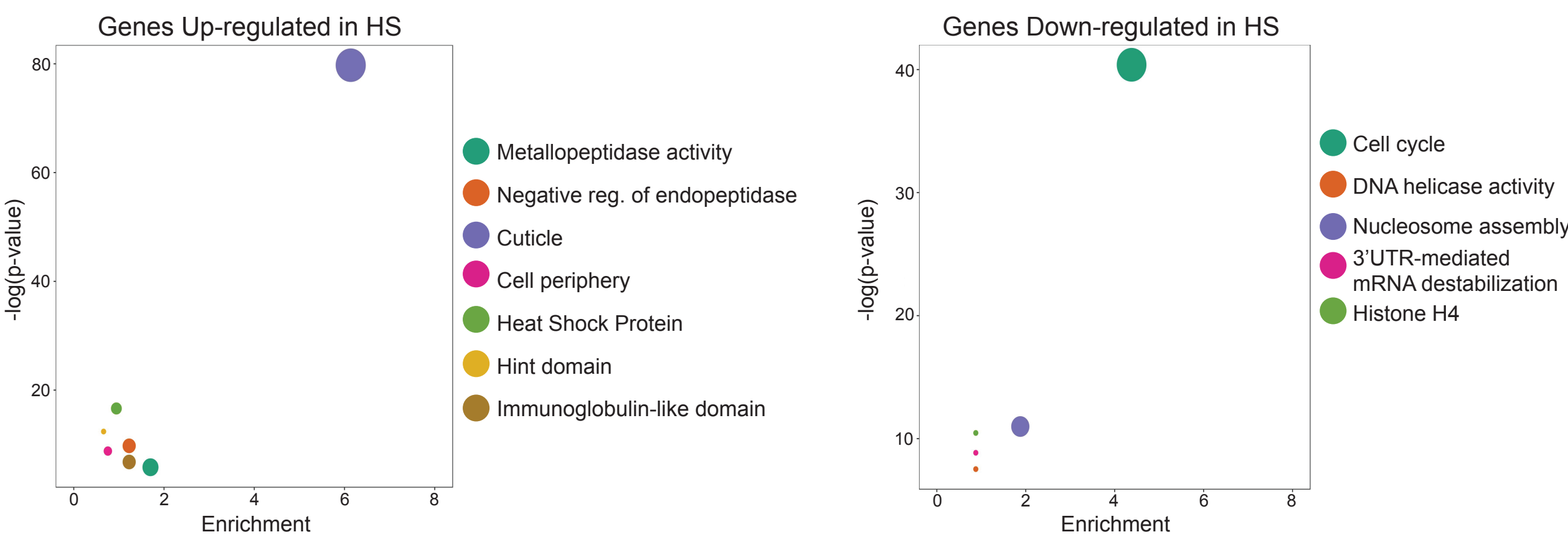

D
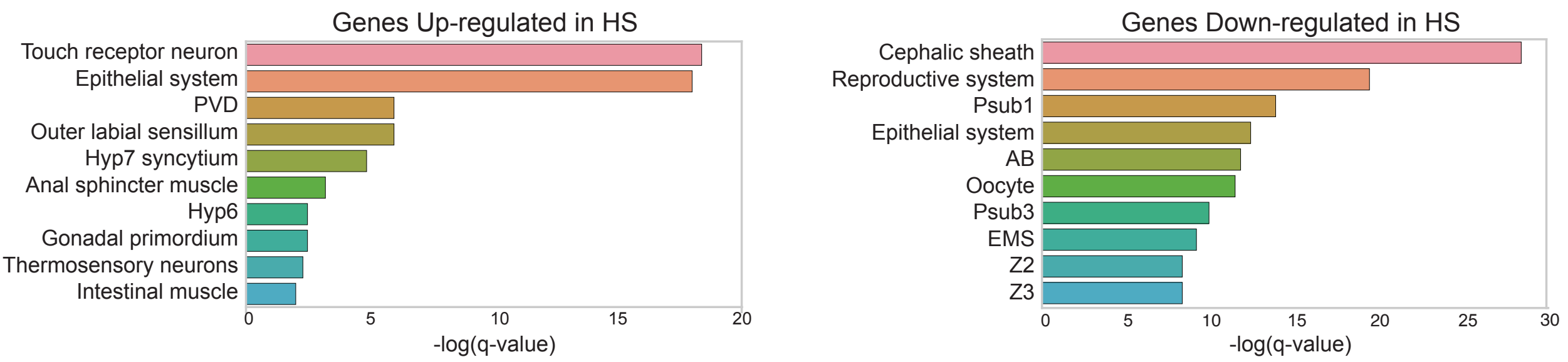
bioRxiv preprint doi: https://doi.org/10.1101/706820; this version posted July 18, 2019. The copyright holder for this preprint (which was not certified by peer review) is the author/funder, who has granted bioRxiv a license to display the preprint in perpetuity. It is made available under

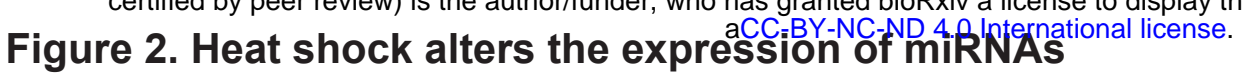

A HS-induced changes in miRNA expression

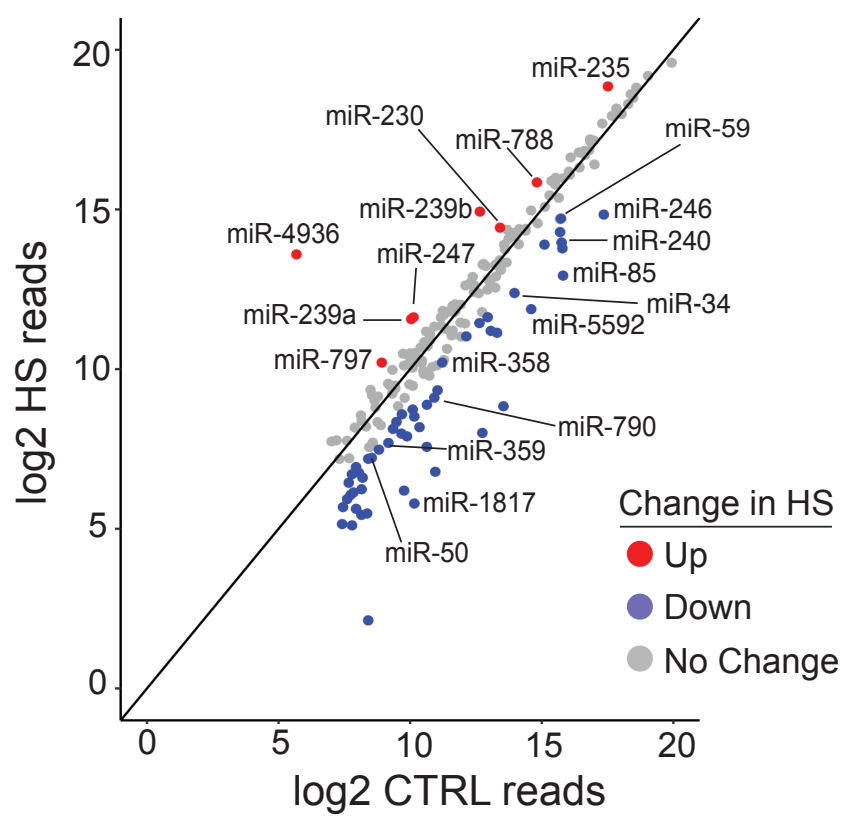

C

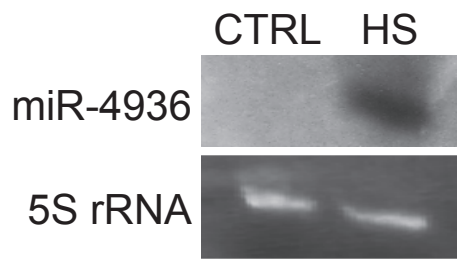

D Overlapping targets of up-regulated miRNAs

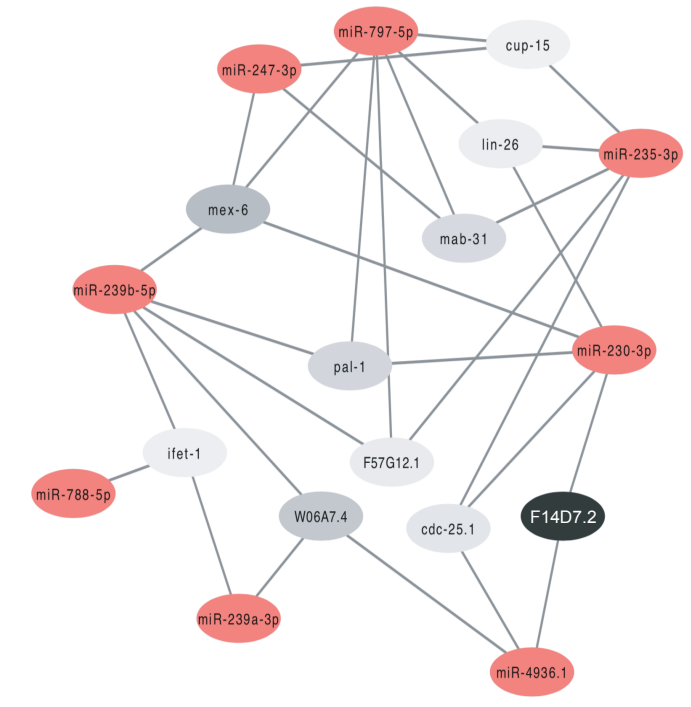

log2 Fold Change

$-5 \quad-1$

mRNA down-regulation in HS
B

miRNAs up-regulated in HS

\begin{tabular}{|c|c|l|}
\hline miRNA & Fold Change & Top GO of targets \\
\hline miR-4936 & 224.3 & Cell Division \\
\hline miR-239b & 4.9 & Reg of Translation \\
\hline miR-239a & 2.9 & Oogenesis \\
\hline miR-247 & 2.8 & mRNA 3'-UTR binding \\
\hline miR-235 & 2.5 & Embryo development \\
\hline miR-797 & 2.4 & Embryo development \\
\hline miR-788 & 2.0 & None \\
\hline miR-230 & 2.0 & mRNA 3'-UTR binding \\
\hline
\end{tabular}

miRNAs down-regulated in HS

\begin{tabular}{|c|c|l|}
\hline miRNA & Fold Change & Top GO of Targets \\
\hline miR-1817 & -20.3 & Molting cycle \\
\hline miR-85 & -7.4 & Muscle contraction \\
\hline miR-5592 & -6.6 & Locomotion \\
\hline miR-246 & -5.7 & Neg reg of endopeptidase \\
\hline miR-240 & -3.5 & Neuron projection dev \\
\hline miR-790 & -3.1 & None \\
\hline miR-34 & -3.0 & Body morphogenesis \\
\hline miR-359 & -2.8 & Axon regeneration \\
\hline miR-50 & -2.3 & Molting cycle \\
\hline miR-59 & -2.0 & Locomotion \\
\hline miR-358 & -2.0 & Body motion \\
\hline
\end{tabular}

E Overlapping targets of down-regulated miRNAs

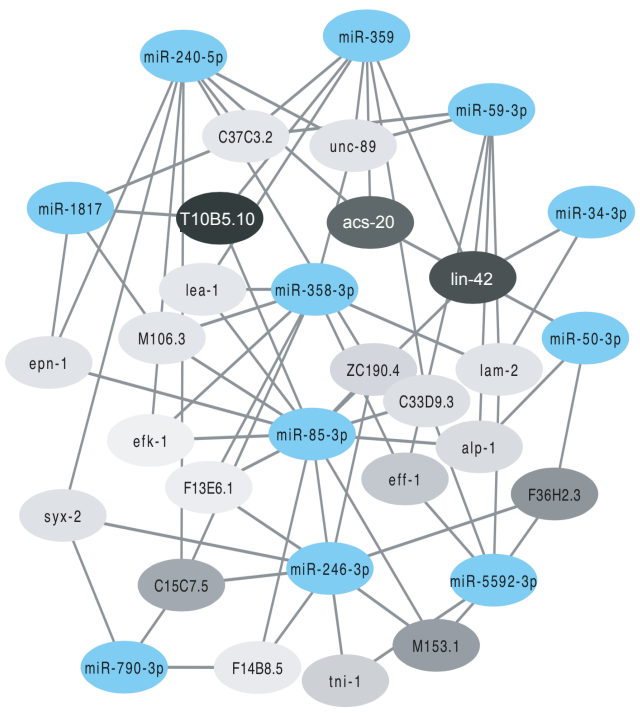

log2 Fold Change

1

mRNA up-regulation in HS 
Figure 3

A

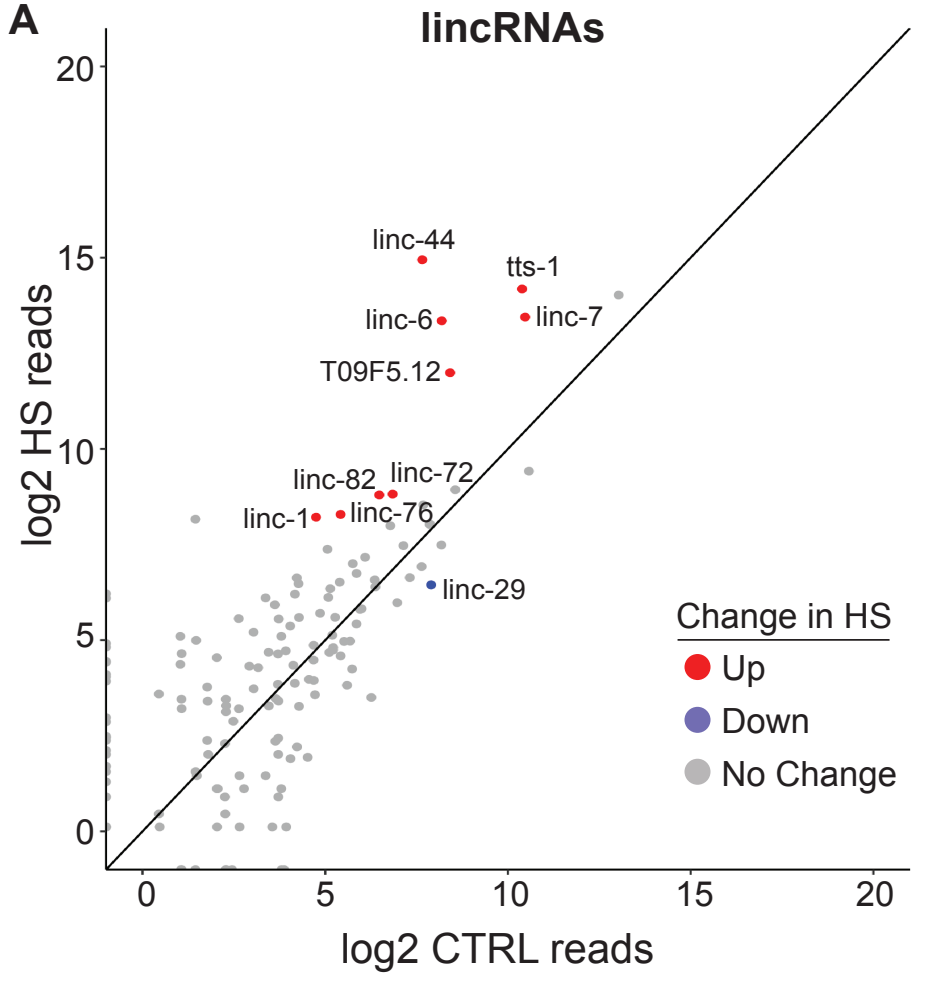

C

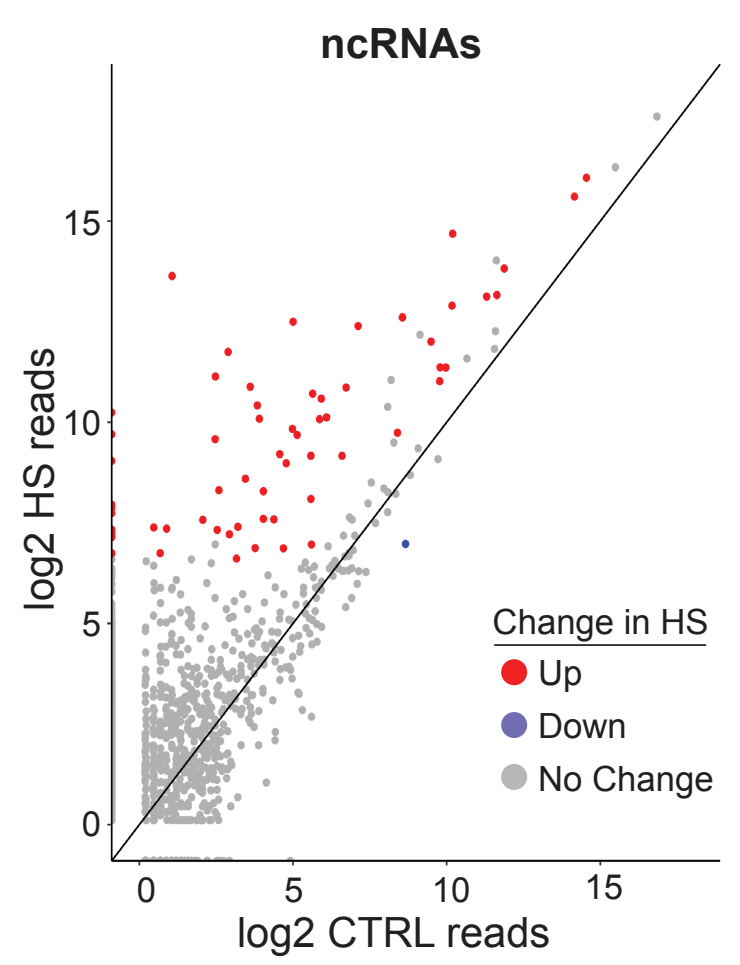

B

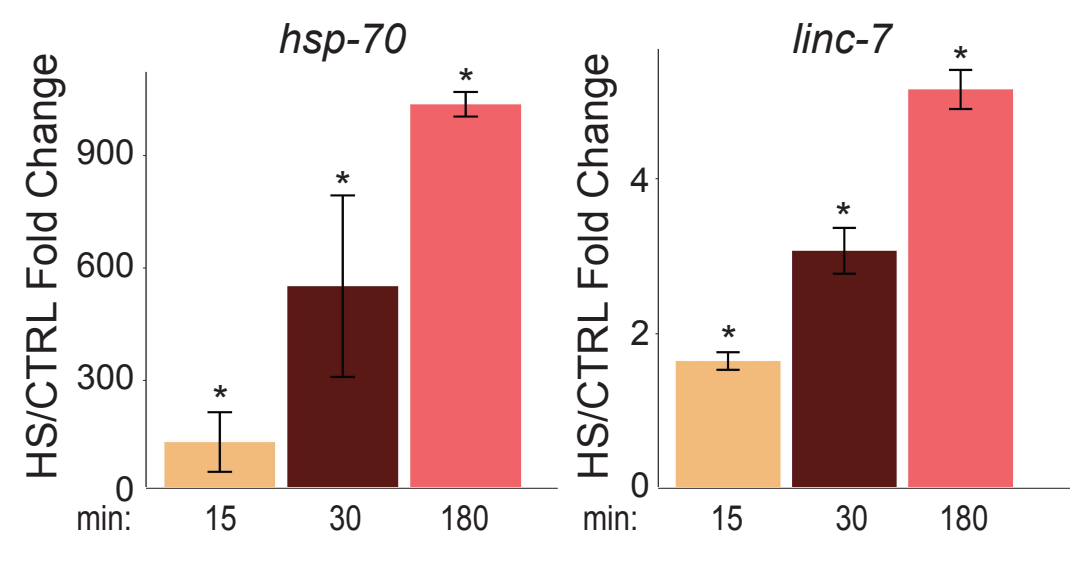

D

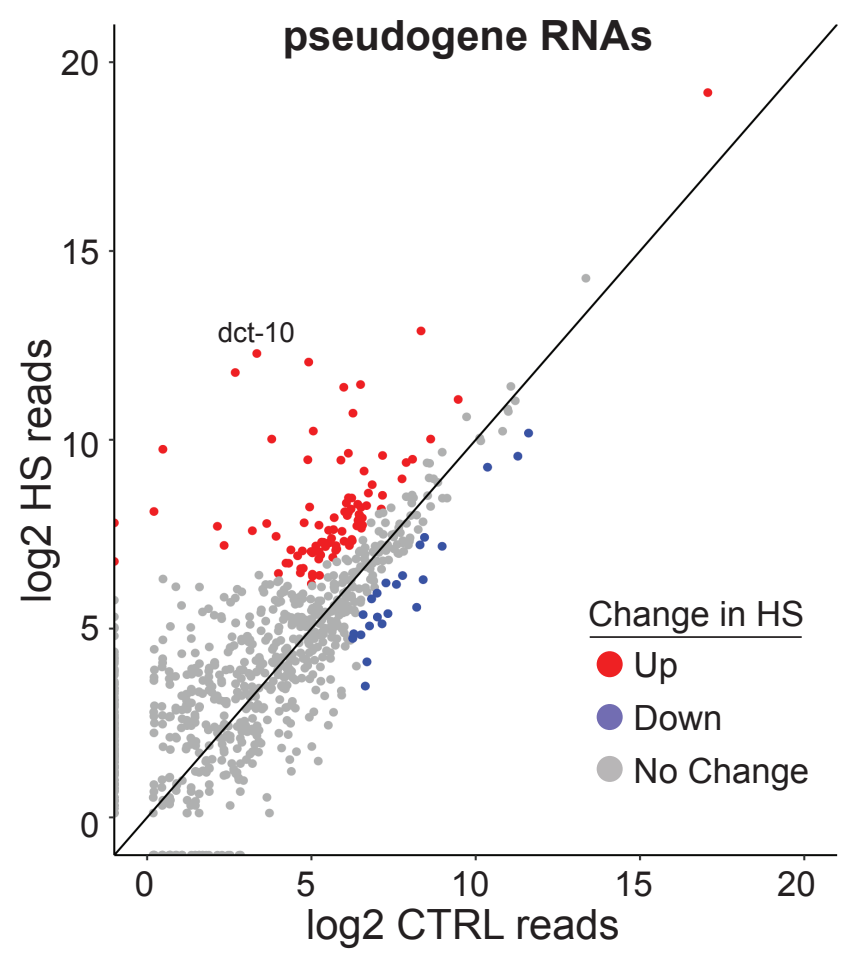


A

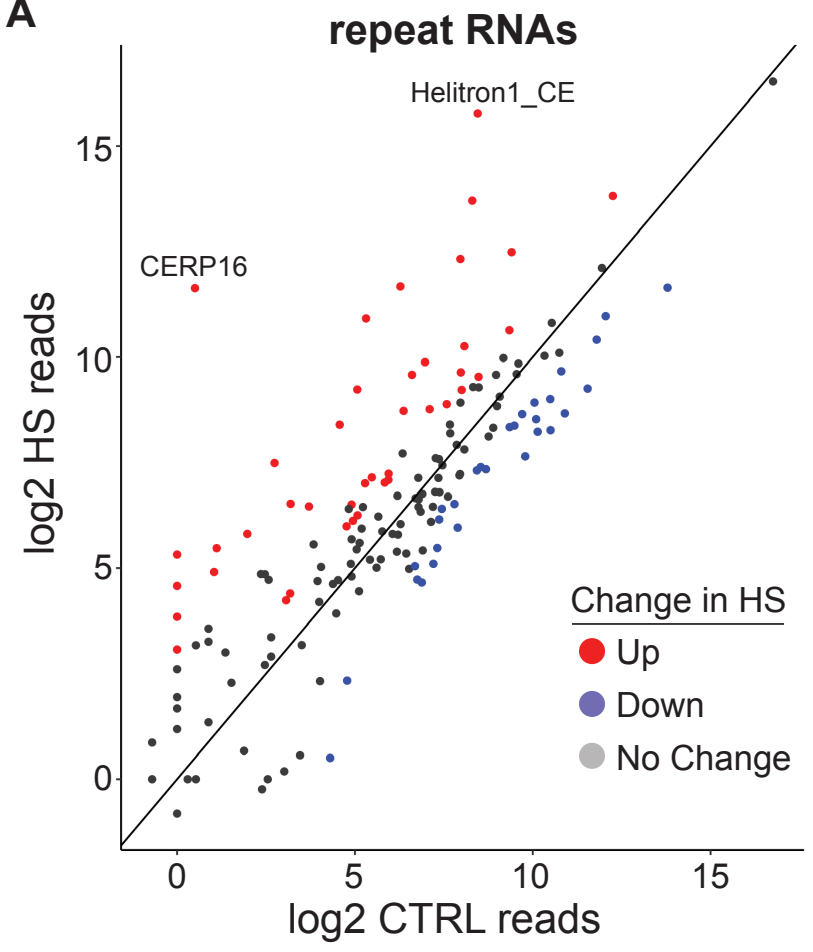

B

\begin{tabular}{|l|c|}
\hline \multicolumn{1}{|c|}{ Repeat } & Fold Up \\
\hline CERP16 & 1698.5 \\
\hline Helitron1_CE & 156.5 \\
\hline Merlin1m_CE & 47.6 \\
\hline TIR43YW1_CE & 41.6 \\
\hline HelitronY1_CE & 41.0 \\
\hline HelitronY1A_CE & 20.2 \\
\hline CER7-LTR_CE & 17.3 \\
\hline RCD1 & 13.6 \\
\hline
\end{tabular}

C

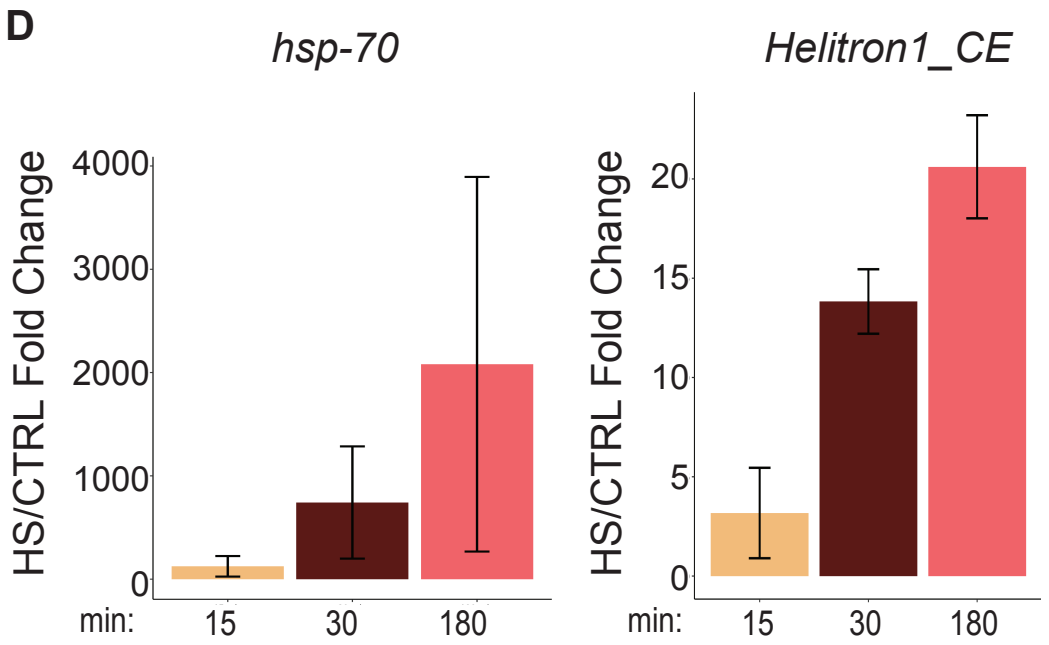


bioRxiv preprint doi: https://doi.org/10.1101/706820; this version posted July 18,2019 . The copyright holder for this preprint (which was not eertifiedAy peer review) is the author/funder, who has granted bioRxiv a license td
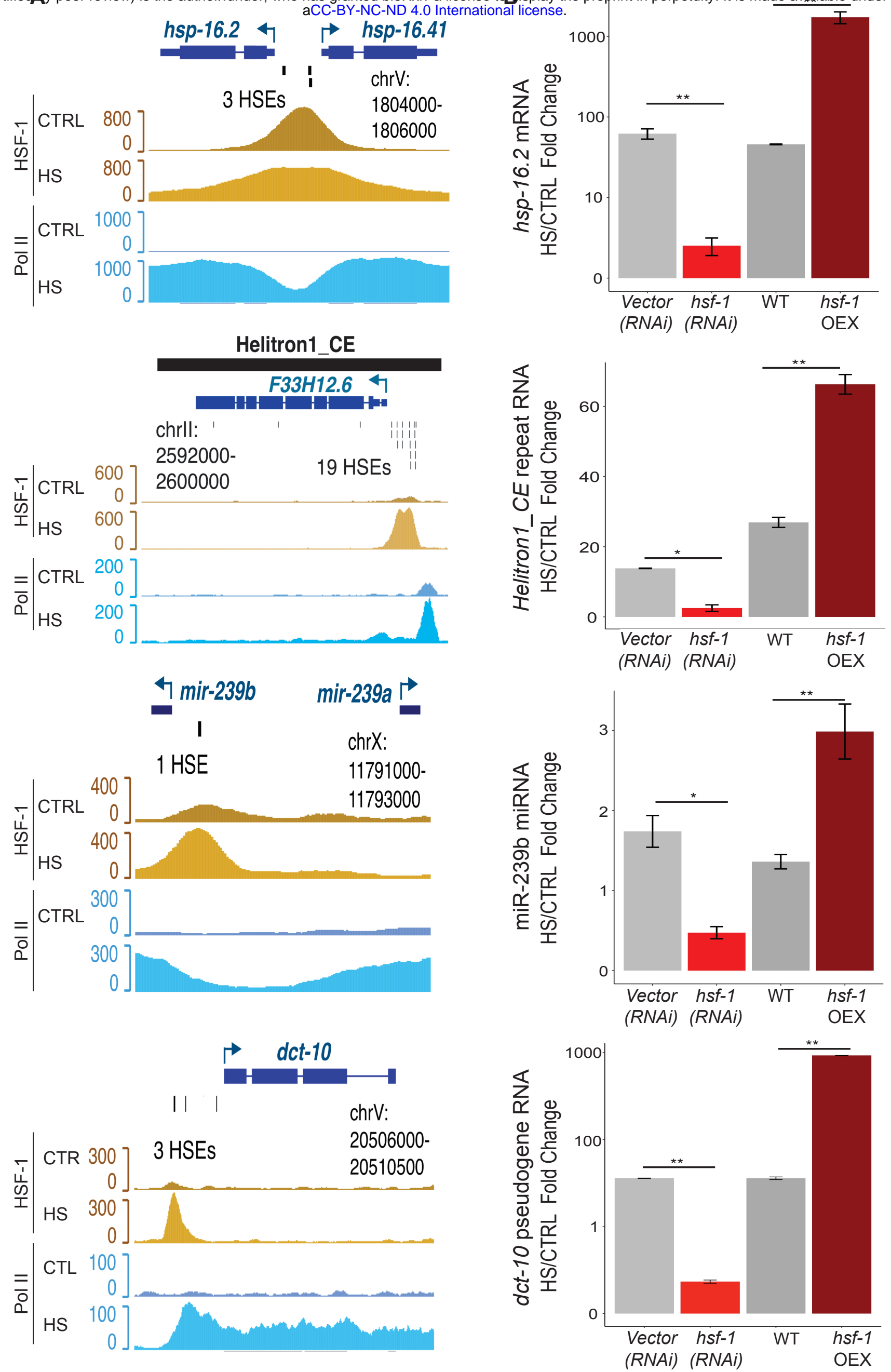